\title{
Heterocedasticidade entre estados para produção de leite em vacas da raça Holandesa, usando métodos bayesianos via amostrador de Gibbs ${ }^{1}$
}

\section{Alencariano José da Silva Falcão ${ }^{2}$, Elias Nunes Martins ${ }^{3}$, Claudio Napolis Costa ${ }^{4}$, Eduardo Shiguero Sakaguti ${ }^{5}$, Josmar Mazucheli ${ }^{6}$}

\author{
${ }_{1}$ Parte da tese de Doutorado apresentada pelo primeiro autor ao Programa de Pós-Graduação em Zootecnia da UEM. \\ 2 PUCPR campus Toledo. Av. da União, 500. Toledo - PR, CEP: 85902-532. \\ 3 Universidade Estadual de Maringá. Av.Colombo, 5790, CEP: 87020-900, Maringá - PR. \\ ${ }^{4}$ Embrapa Gado de Leite. R. Eugênio do Nascimento, 610, CEP: 36038-330, Juiz de Fora - MG. \\ ${ }^{5}$ Departamento de Zootecnia da Universidade Estadual de Maringá. \\ ${ }^{6}$ Departamento de Matemática e Estatística da Universidade Estadual de Maringá.
}

RESUMO - Registros de produção de leite ajustados para 305 dias de lactação (PL305) em vacas da raça Holandesa que pariram entre 1980 e 1993 foram utilizados para investigar a existência de heterogeneidade de variância e a interação genótipo $\times$ ambiente. Supondo-se variâncias heterogêneas, as PL305 nos estados de Minas Gerais, São Paulo, Paraná, Santa Catarina e Rio Grande do Sul foram tratadas como características diferentes. Admitindo-se homogeneidade de variância, a PL305 foi analisada segundo um modelo unicaracter. Os componentes de (co)variância e os parâmetros genéticos foram estimados utilizando-se métodos Bayesianos, via amostrador de Gibbs (GS), sob um modelo animal que incluiu os efeitos fixos de estação de parto, grupo genético, ordem de parição e classes rebanho-ano de parto e os efeitos genéticos aditivos e de ambiente permanente. A monitoração de convergência das distribuições das cadeias foi realizada pelo método de Heidelberg \& Welch (1983). As estimativas dos componentes de variância e de herdabilidade foram obtidas com grande precisão na análise unicaracter. A média e o desvio-padrão (DP) a posteriori da herdabilidade foram $0,278 \pm 0,012$. Na análise multicaracter, as estimativas mais precisas da (co)variância genética foram as obtidas em São Paulo e Paraná. As médias e os desvios-padrão a posteriori de herdabilidade para Minas Gerais, São Paulo, Paraná, Santa Catarina e Rio Grande do Sul, foram 0,280 $\pm 0,021$, $0,233 \pm 0,015,0,280 \pm 0,012,0,393 \pm 0,026$ e $0,382 \pm 0,022$, respectivamente. A baixa magnitude das correlações genéticas entre os estados $(0,070$ a 0,364$)$ sugere a existência da interação genótipo $\times$ ambiente, logo, as PL305 em cada estado devem ser tratadas como características diferentes. O maior valor de correlação genética foi encontrado em São Paulo e Paraná. As variâncias genética e residual para PL305 foram significativamente diferentes entre a maioria dos estados.

Palavras-chave: componentes de variância, convergência, herdabilidade, inferência Bayesiana, interação genótipo $\times$ ambiente

\section{An evaluation of heteroscedasticity of variances of milk yield of Holstein cattle in different states using Bayesian inference via Gibbs sampler}

ABSTRACT - Adjusted for 305 days milk yield records of Holstein cows calving from 1980 to 1993 in the states of MG, $\mathrm{SP}, \mathrm{PR}, \mathrm{SC}$, and RS were used to investigate heterogeneity of variance and to evaluate the genotype by environment interaction. Milk production from each State was considered as a different trait and variances were assumed heterogeneous. Milk production was also analyzed using a single-trait model assuming homogeneity of variance. (Co)variance components and genetic parameters were estimated by Bayesian inference, via Gibbs sampler (GS), using a model which included season of calving, genetic group, herd-year of calving and parity as fixed effects and animal additive genetic, permanent environmental and residual as random effects. Convergence of the GS chain to the stationary distribution was diagnosed using the method described by Heidelberg \& Welch (1983). The posterior precision of the variance components and the heritability were high in the singletrait analysis. Posterior mean and standard deviation (SD) of heritability of milk yield were $0,278 \pm 0,012$. For the multipletrait analysis, posterior precisions of the (co)variance components were larger for SP and PR states. Posterior means and standard errors of heritability for MG, SP, PR, SC, and RS were $0.280 \pm 0.021,0.233 \pm 0.015,0.280 \pm 0.012,0.393 \pm 0.026$, and $0.382 \pm 0.022$, respectively. Genetic correlations for milk yield between the five states were very low and ranged from 0.070 to 0.364 , suggesting the presence of genotype by environment interaction. Differences in genetic and residual variances of milk yield among the states indicate it would be necessary to account for heterogeneous variances in genetic evaluations.

Key Words: Bayesian inference, convergence criterion, heritability, variance components, genotype $\times$ environment interaction 


\section{Introdução}

O progresso genético nos sistemas de produção animal exige avaliações genéticas acuradas dos animais, seguidas da seleção dos melhores indivíduos que serão pais da próxima geração. Na maioria dos programas de melhoramento de gado de leite, a seleção é baseada na Melhor Predição Linear Não-Viesada (BLUP) do valor genético, obtida pela metodologia de modelos mistos. Embora tenha havido ganho genético substancial nos sistemas que utilizam esta metodologia, este ganho não é igual ao teoricamente possível. De acordo com DeStefano (1994), uma razão para que o atual progresso genético alcançado nos rebanhos não seja igual ao teoricamente esperado é que as pressuposições nas quais as atuais avaliações genéticas se baseiam têm sido violadas. Entre estas premissas, encontra-se a homogeneidade de variância.

Vários estudos comprovam a existência de heterogeneidade de variâncias genética, residual, fenotípica e de ambiente permanente para características de produção, em relação à região, ao nível de produção e ao rebanho, entre outros fatores (Hill et al., 1983; Famula, 1989; Boldman \& Freeman, 1990; Costa, 1999). Portanto, nesses casos, a premissa de homocedasticidade das variâncias não tem sustentação e admiti-las nos modelos pode implicar na introdução de viés nas avaliações dos animais.

Um problema de grande relevância, quanto à heterogeneidade de variância na avaliação genética de bovinos leiteiros, está no fato de que os animais acima da média, nos rebanhos que apresentam maior variação podem ter seus valores genéticos superestimados (Brotherstone \& Hill, 1986; Everett et al., 1982). Portanto, grande parte dos reprodutores e das matrizes pode ser selecionada de rebanhos que apresentam maior variabilidade. A avaliação de reprodutores pode constituir-se em problema se os touros não são usados homogeneamente entre rebanhos.

A maioria dos estudos sobre a presença de heterogeneidade de variância relata que as variâncias genética e ambiental aumentam com a elevação da produção média de leite no rebanho (Mirande \& Van Vleck, 1985; De Veer \& Van Vleck, 1987; Boldman \& Freeman, 1990), afetando diretamente as estimativas de coeficientes de herdabilidade. Vários estudos em rebanhos da raça Holandesa mostraram tendência de aumento nas herdabilidades com o acréscimo na média de produção (Hill et al., 1983; Boldman \& Freeman, 1990; Dong \& Mao, 1990; Valencia et al., 1998).

Para estimar os componentes de (co)variância, De Veer \& Van Vleck (1987) estratificaram os dados de produção de leite em três níveis (baixo, médio e alto), considerados três características. Entretanto, segundo Famula (1989), a determinação da existência de heterogeneidade de variância entre grupos de rebanhos, baseada na estratificação dos dados pela produção média de leite do rebanho, equivale a selecionar pelas médias dos rebanhos, o que possibilita estimativas viesadas das variâncias genética e residual.

Henderson (1984) relatou que suas equações de modelos mistos (EMM) podem ser modificadas para considerar as variâncias genéticas ou residuais que diferem entre ambientes. Uma abordagem é a modelagem para características múltiplas, em que o valor genético em cada rebanho ou os dados estratificados por produções são tratados como características distintas.

Os preditores BLUP para os efeitos aleatórios, resultantes das EMM, podem convenientemente considerar as diferenças de variâncias dentro das subclasses. Porém, esse método pode exigir a estimação de grande número de componentes de variância, com pouca informação contribuindo para cada componente. Nessas situações, os métodos de verossimilhança que dependem de uma justificativa assimptótica podem falhar em prover estimativas suficientemente acuradas (Gianola, 1986; Weigel et al., 1993).

Falconer (1952) introduziu o conceito de uma correlação genética entre o desempenho em dois ambientes como sendo duas características diferentes e utilizou a razão entre as respostas indireta e direta à seleção para determinar o melhor ambiente para seleção. Robertson et al. (1960) identificou a necessidade de se reconhecer se a herdabilidade difere entre ambientes e se a classificação dos touros é afetada por essas diferenças.

Hill et al. (1983) estimaram parâmetros genéticos para produção de leite em rebanhos da raça Holandesa. Os rebanhos foram divididos em dois grupos, de acordo com os níveis de produção (baixa e alta). As estimativas de herdabilidade nos rebanhos de baixa e alta produção de leite foram 0,24 e 0,30 , respectivamente. As correlações genéticas entre os níveis de alta e baixa produção foram próximas da unidade e as vacas de maiores méritos foram encontradas nos rebanhos de maiores variâncias. No Brasil, Torres et al. (1999) analisaram registros de primeira lactação de vacas Holandesas, os quais foram estratificados em rebanhos conforme o desvio-padrão fenotípico e submetidos às transformações logarítmica, raiz quadrada, padronização e divisão pelo desvio-padrão fenotípico (DPF) da classe analisada. As estimativas de herdabilidade nas escalas original, logarítmica, raiz quadrada e padronizada e a divisão pelo DPF foram 0,$27 ; 0,34 ; 0,30 ; 0,27 \mathrm{e} 0,27$, respectivamente, para classe de desvio-padrão baixo. Os valores correspondentes para classes de desvios-padrão médio e alto foram 0,35 ; 
0,$40 ; 0,37 ; 0,35$ e 0,35 e 0,$30 ; 0,36 ; 0,32 ; 0,30$ e 0,30 , respectivamente. Costa (1999) investigou a heterogeneidade de variância para produções de leite e gordura em vacas da raça Holandesa utilizando registros de primeira lactação ajustada para 305 dias e para idade adulta. A estimativa de herdabilidade para produção de leite dos rebanhos pertencentes à classe de baixo desvio-padrão fenotípico foi superior à estimada para os rebanhos da classe de alto desviopadrão $(0,30$ e 0,22 , respectivamente $)$.

No Brasil, os rebanhos de vacas da raça Holandesa estão situados, em sua maioria, nas Regiões Sul e Sudeste, de modo que diferentes condições de clima, alimentação e manejo podem ser fontes de heterogeneidade de variância e/ou interação genótipo $\times$ ambiente. Assim, os objetivos neste trabalho foram obter estimativas de componentes de (co)variância e parâmetros genéticos para produção de leite, estimar a magnitude das correlações genéticas entre os estados e investigar a existência da heterogeneidade de variância da produção de leite entre os estados.

\section{Material e Métodos}

Foram disponibilizados 172.051 registros de lactações de 100.178 vacas, cujas parições ocorreram entre os anos de 1980 e 1993, distribuídas em 1.626 rebanhos. Essas informações foram geradas dentro do programa oficial de controle leiteiro da raça Holandesa, conduzido por associações e núcleos de criadores de Minas Gerais, São Paulo, Paraná, Santa Catarina e Rio Grande do Sul, integrantes do Arquivo Zootécnico Nacional, gerenciado pela EMBRAPA Gado de Leite, em Juiz de Fora-MG.

O conjunto de dados original foi editado com uso do sistema SAS ${ }^{\circledR}$ (SAS, 2001), objetivando eliminar os registros com erros, as observações incompletas e as causas de secagem anormais e verificar inconsistências nos registros de pedigree e informações sobre número de lactações e idade ao parto. Foram eliminadas as classes de rebanho-ano de parição com menos de 15 observações, visando melhorar a estrutura dos dados. Após todas as restrições impostas ao conjunto original de dados, obteve-se um conjunto final (108.702 registros de produção de leite) com lactações entre 150 e 450 dias de duração e produção entre 1.000 e $13.000 \mathrm{~kg}$ de leite. Na Tabela 1 constam as distribuições das lactações por ordem de parto e estado.

Os seguintes limites de idade, em meses, dentro de ordem de parto (OP) foram estabelecidos: $20 \leq$ OP $1 \leq 60 ; 30$ $\leq \mathrm{OP} 2 \leq 78 ; 42 \leq$ OP $3 \leq 96 ; 54 \leq$ OP $4 \leq 116$ e $66 \leq$ OP $5 \leq 134$. As informações da origem e ascendência das vacas possibilitaram classificá-las em dois grupos genéticos: no primeiro grupo, estão incluídas as vacas Holandesas puras de origem e, no segundo grupo, aquelas com composição genética superior ou igual a 31/32 da raça Holandesa. Foram definidas duas estações de parição: uma de abril a setembro e outra de outubro e março.

A estimação dos componentes de variância e da herdabilidade para produção de leite ajustada para 305 dias de lactação (PL305), supondo-se homogeneidade e variância, foi realizada segundo um modelo animal unicaracter, descrito na forma matricial em [1]. Desta forma, as produções de leite nos diferentes estados foram consideradas como única característica. A implementação dos princípios bayesianos na estimação dos componentes de (co)variância foi realizada por meio dos programas do sistema MTGSAM (Multiple Trait Gibbs Sampling in Animal Model), desenvolvido por Van Tassell \& Van Vleck (1995).

$$
y=X \beta+Z a+W p+e
$$

em que $y$ é o vetor das produções de leite (PL305); $\beta$, o vetor dos efeitos fixos da subclasse rebanho-ano de parição, estação de parto, grupo genético e ordem de parto com componentes linear e quadrático; $a$, o vetor dos efeitos aleatórios dos valores genéticos diretos; $p$, o vetor das contribuições de ambiente permanente; $e$, o vetor de resíduos; e $X, Z$ e $W$, as matrizes de incidência dos efeitos fixos, genéticos diretos e de ambiente permanente, respectivamente.

Admitiu-se que $y, a, p$ e $e$ possuem distribuição conjunta normal multivariada, como descrito abaixo:

$\left[\begin{array}{c}y \\ a \\ p \\ e\end{array}\right] \sim N M V\left\{\left[\begin{array}{c}X \beta \\ \phi \\ \phi \\ \phi\end{array}\right],\left[\begin{array}{cccc}Z G Z^{\prime}+W P W^{\prime}+R & Z G & W P & R \\ G Z^{\prime} & G & \phi & \phi \\ P W^{\prime} & \phi & P & \phi \\ R & \phi & \phi & R\end{array}\right]\right\}$,

em que $G=A \sigma_{a}^{2}, P=I_{p} \sigma_{p}^{2}$ e $R=I \sigma_{e}^{2}$, de modo que $A$ é a matriz de coeficientes de parentesco entre os animais, de ordem $m ; \sigma_{a}^{2}$, a variância genética aditiva; $\sigma_{p}^{2}$, a variância de efeito permanente de ambiente, associado às vacas; $\sigma_{e}^{2}$, a variância de efeito temporário de ambiente; $I$, uma matrizidentidade de ordem $n ; I_{P}$, uma matrizidentidade de ordem $p ; n$, o número total de lactações; $p$, o número de vacas com registro de lactação; $Z$ e $W$, as matrizes de incidência dos valores genéticos e dos efeitos de ambiente permanentes, de ordens $n \times m$ e $n \times p$, respectivamente.

Para estimação dos componentes de (co)variância e dos parâmetros genéticos, supondo-se heterogeneidade de variância, a PL305 em cada um dos cinco estados foi tratada como uma característica diferente. Neste caso, as 
Tabela 1 - Distribuições das lactações por ordem de parto e estado

Table 1 - Number of records according to parity, and state

\begin{tabular}{lcccccc}
\hline $\begin{array}{l}\text { Estado } \\
\text { State }\end{array}$ & \multicolumn{5}{c}{$\begin{array}{c}\text { Ordem de parto } \\
\text { Parity }\end{array}$} & Total \\
& \multicolumn{7}{c}{2} & 3 & 4 & 5 & \\
\hline MG & 2870 & 2456 & 1755 & 1079 & 570 & 8730 \\
SP & 9858 & 7549 & 4849 & 3163 & 1807 & 27226 \\
PR & 24908 & 16161 & 11526 & 7580 & 4640 & 64815 \\
SC & 1457 & 988 & 625 & 313 & 169 & 3552 \\
RS & 1426 & 1104 & 850 & 605 & 394 & 4379 \\
Total & 40519 & 28258 & 19605 & 12740 & 7580 & 108702
\end{tabular}

definições para os elementos do modelo animal e da distribuição conjunta de $Y, a, p$ e $e$ são as seguintes:

$y=\left[\begin{array}{c}y_{1} \\ y_{2} \\ \vdots \\ y_{5}\end{array}\right]=\left[\begin{array}{cccc}X_{1} & 0 & \cdots & 0 \\ 0 & X_{2} & \cdots & 0 \\ \vdots & \vdots & \ddots & \vdots \\ 0 & 0 & \cdots & X_{5}\end{array}\right] \cdot\left[\begin{array}{c}\beta_{1} \\ \beta_{2} \\ \vdots \\ \beta_{5}\end{array}\right]+\left[\begin{array}{cccc}Z_{1} & 0 & \cdots & 0 \\ 0 & Z_{2} & \cdots & 0 \\ \vdots & \vdots & \ddots & \vdots \\ 0 & 0 & \cdots & Z_{5}\end{array}\right] \cdot\left[\begin{array}{c}a_{1} \\ a_{2} \\ \vdots \\ a_{5}\end{array}\right]+\left[\begin{array}{cccc}W_{1} & 0 & \cdots & 0 \\ 0 & W_{2} & \cdots & 0 \\ \vdots & \vdots & \ddots & \vdots \\ 0 & 0 & \cdots & W_{5}\end{array}\right] \cdot\left[\begin{array}{c}p_{1} \\ p_{2} \\ \vdots \\ p_{5}\end{array}\right]+\left[\begin{array}{c}e_{1} \\ e_{2} \\ \vdots \\ e_{5}\end{array}\right]$

em que $y_{1}, y_{2}, \ldots, y_{5}$ são os vetores de observações, PL305; $\beta_{1}, \beta_{2}, \ldots, \beta_{5}$, os vetores dos efeitos fixos; $a_{1}, a_{2}, \ldots, a_{5}$, os vetores dos efeitos genéticos diretos; $p_{1}, p_{2}, \ldots, p_{5}$, os vetores dos efeitos permanentes de ambiente; $X_{1}, X_{2}, \ldots, X_{5}$, as matrizes de incidência dos efeitos fixos; $Z_{1}, Z_{2}, \ldots, Z_{5}$, as matrizes de incidência dos efeitos genéticos diretos; $W_{1}, W_{2}, \ldots W_{5}$, as matrizes de incidência dos efeitos permanentes de ambiente; $e_{1}, e_{2}, \ldots, e_{5}$, os vetores dos erros aleatórios; os índices $1,2, \ldots 5$, os estados de $\mathrm{MG}, \mathrm{SP}, \mathrm{PR}, \mathrm{SC}$ e RS, respectivamente; $G=G_{0} \otimes A$, em que $G_{0}$ é a matriz de variância e covariância genéticas dos $i$ estados determinada por

$G_{0}=\left[\begin{array}{cccc}\sigma_{a_{1}}^{2} & \sigma_{a_{1} a_{2}} & \cdots & \sigma_{a_{1} a_{5}} \\ \sigma_{a_{2} a_{1}} & \sigma_{a_{2}}^{2} & \cdots & \sigma_{a_{2} a_{5}} \\ \vdots & \vdots & \ddots & \vdots \\ \sigma_{a_{5} a_{1}} & \sigma_{a_{5} a_{2}} & \cdots & \sigma_{a_{5}}^{2}\end{array}\right]$

sendo $\sigma_{a_{i}}^{2}$ a variância genética aditiva do estado $i$ e $\sigma_{a_{i j}}$ a covariância genética entre os estados $i$ e $j ; P=\oplus_{i=1} I_{i} \sigma_{p_{i}}^{2}$, em que $i_{5}$ identifica o estado onde o animal foi observado; $R=\bigoplus_{i=1}^{5} I_{i} \sigma_{e_{i}}^{2}$, em que $i$ identifica o estado onde o animal foi observado.

As distribuições a priori foram definidas como a seguir: para os efeitos fixos, assumiu-se uma distribuição vaga, $p(\beta) \propto$ cons tan te. Aos componentes de (co)variância genética, atribuiu-se uma distribuição de Wishart invertida, $G \sim I W\left(G_{0}^{*}, v_{g}\right)$, cuja densidade é determinada por $f\left(G \mid G_{0}^{*}, v_{g}\right) \propto(G)^{-1 / 2\left(v_{g}+m_{g}+l\right)} \cdot e^{-1 / 2 t r\left[\left[G_{0}^{*}\right)^{-l} G^{-l}\right]}$, em que $m_{g}$ é a ordem da matriz $G$ e $G_{0}^{*}$ é a matriz de parâmetro escala da distribuição de $G$. Para os componentes de variância de ambiente permanente, considerou-se que cada elemento $\sigma_{p_{i}}^{2}$ tem distribuição de gama invertida, $\sigma_{p_{i}}^{2} \sim \Gamma I\left(p_{a}, v_{d}\right)$, cuja densidade é $f\left(\sigma_{p_{\theta_{i}}}^{2} \mid p_{o_{l}}, v_{p_{i}}\right) \propto\left(\sigma_{p_{\theta_{i}}}^{2}\right)^{-1 / 2\left(v_{p_{i}}+2\right)^{p_{i}}} \cdot e^{-1 / 2\left(p_{\theta_{i}} \sigma_{p_{\theta_{i}}}^{-}\right)}$, em que $p_{o_{i}}$ é o parâmetro escala da distribuição de $\sigma_{p_{0_{i}}}^{2}$. Para os componentes de variância residual, foi assumido que cada elemento $\sigma_{e_{i}}^{2}$ tem distribuição de gama invertida, $\sigma_{e_{i}}^{2} \sim \Gamma I\left(s_{o}, v_{r}\right)$, cuja densidade é $f\left(\sigma_{e_{i}}^{2} \mid s_{0_{i}}, v_{r_{i}}\right) \propto\left(\sigma_{e_{i}}^{2}\right)^{-1 / 2\left(v_{r_{i}}+2\right)} \cdot e^{-1 / 2\left(s_{\theta_{i}} \sigma_{e_{i}}^{-2}\right)}$, em que $s_{o_{i}}$ é o parâmetro escala da distribuição de $\sigma_{e_{i}}^{2} . v_{g}, v_{p_{i}}$ e $v_{r_{i}}$ correspondem aos números de graus de liberdade das distribuições de $G, \sigma_{p_{i}}^{2}$ e $\sigma_{e_{i}}^{2}$, respectivamente. Os valores atribuídos aos parâmetros de forma e posição para a distribuição de Wishart invertida foram 7 e as estimativas REML, respectivamente. Para as distribuições de gama invertida, esses valores foram 3 e as estimativas REML. A densidade conjunta a posteriori é descrita como:

$$
\begin{gathered}
f\left(\beta, a, G, R / y, v_{g}, G_{0}, v_{r_{i}}, s_{0_{l}}, \ldots, v_{r_{r}}, s_{0_{6}}\right) \\
\propto|R|^{-1 / 2} \cdot \exp \left\{-1 / 2(y-X \beta-Z a-W p)^{\prime} R^{-1}(y-X \beta-Z a-W p)\right\} . \\
|G|^{-1 / 2\left(n+v_{g}+d_{0}+1\right)} \cdot \exp \left\{-1 / 2\left[a^{\prime}\left(G^{-1} \otimes A^{-1}\right) a+\operatorname{tr}\left(v_{g}^{*} G_{0} G^{-1}\right)\right]\right\} . \\
\prod_{i=1}^{5}\left[\left|\sigma_{p_{i}}^{2}\right|^{-1 / 2\left(n_{i}+v_{p_{i}}+1\right)} \cdot \exp \left\{-1 / 2\left(a^{\prime}\left(\sigma_{p_{i}}^{-2} \otimes I_{n_{i}}\right) a\right)+\left(v_{p_{i}}^{*} d_{0_{i}} \sigma_{p_{i}}^{-2}\right)\right\}\right] . \\
\prod_{i=1}^{5}\left(\sigma_{e_{i}}^{2}\right)^{-1 / 2\left(v_{r_{i}}+2\right)} \cdot \exp \left\{-\frac{v_{r_{i}}^{*}}{2} s_{0_{i}} \sigma_{e_{i}}^{-2}\right\} .
\end{gathered}
$$

em que $n=$ número de animais.

Para avaliar os laços genéticos entre pares de estados, foi utilizado o conceito de similaridade genética, definido por Rekaya et al. (1999) como a razão entre o número de filhas dos touros comuns entre o par de estados e o número de filhas de todos os touros utilizados no respectivo par de estados. Na Tabela 2 consta a similaridade genética entre os cinco estados e o número de touros comuns com mais de quatro filhas entre cada par de estados.

Para a análise unicaracter, foram geradas, para cada parâmetro, cadeias de Gibbs de 80.000 iterações, com um

Tabela 2 - Similaridade genética (acima da diagonal) e número de touros comuns (abaixo da diagonal) com mais de quatro filhas usados entre os cinco estados

Table 2 - Genetic similarity (above diagonal) and number of bulls in common (below diagonal) with more than four daughters used between five states

\begin{tabular}{lccccc}
\hline $\begin{array}{l}\text { Estado } \\
\text { State }\end{array}$ & MG & SP & PR & SC & RS \\
\hline MG & & 0,56 & 0,68 & 0,30 & 0,45 \\
SP & 356 & & 0,62 & 0,26 & 0,46 \\
PR & 357 & 514 & & 0,35 & 0,56 \\
SC & 66 & 95 & 98 & & 0,29 \\
RS & 162 & 223 & 237 & 64 & \\
\hline
\end{tabular}


descarte inicial de 30.000 iterações e intervalo de amostragem a cada 50 iterações. Entretanto, para a análise multicaracter, em razão da maior complexidade do modelo, foi estabelecida outra estratégia para implementar o GS. Inicialmente, foram geradas cadeias de 600.000 iterações para cada parâmetro, sem descarte inicial e intervalo de amostragem a cada duas iterações. A seguir, as cadeias foram submetidas ao diagnóstico de convergência, a fim de verificar as respectivas estacionariedades. Para que todas as cadeias convergissem, efetuou-se um descarte inicial de 100.000 iterações, com intervalo de amostragem a cada 100 iterações, resultando em cadeias de tamanho 2.000 para todos os parâmetros.

A convergência das cadeias de Gibbs para distribuições estacionárias dos componentes de variância foi diagnosticada utilizando-se o método de Heidelberg \& Welch (1983), implementado na biblioteca CODA (Convergence Diagnosis and Output Analysis) versão 0.4, desenvolvido por Cowles et al. (1995). Tanto nas análises unicaracter como na multicaracter as cadeias de GS atingiram a convergência.

A estatística $z$ foi aplicada para avaliar a homogeneidade das variâncias genética e residual entre os estados, assumindo a normalidade para suas distribuições a posteriori. Para o cálculo de $z$, foram utilizadas as médias e os respectivos desvios-padrão a posteriori de cada parâmetro.

\section{Resultados e Discussão}

As médias observadas para produção de leite ajustada para 305 dias de lactação (PL305), com seus respectivos desvios-padrão, nos estados de Minas Gerais, São Paulo, Paraná, Santa Catarina e Rio Grande do Sul foram 4.696 \pm 1543 , $5.035 \pm 1590,6.291 \pm 1508,4.832 \pm 1502$ e $4.781 \pm 1551 \mathrm{~kg}$, respectivamente.
$\mathrm{Na}$ Tabela 3 encontram-se as médias a posteriori e os respectivos desvios-padrão (DP) e intervalos de credibilidade dos componentes de variância genética, da proporção da variância de ambiente permanente e de herdabilidade para PL305, nos cinco estados, obtidas pelo modelo unicaracter.

$\mathrm{Na}$ análise unicaracter, as estimativas dos componentes de variância e de herdabilidade foram obtidas com grande precisão, em virtude do grande número de dados, como indicado pela pequena magnitude dos DP a posteriori e pelas amplitudes dos intervalos de credibilidade $a$ posteriori, entre 2,5 e $97,5 \%$.

A média a posteriori de herdabilidade para PL305, sob o modelo unicaracter, está dentro do intervalo observado na literatura (Dong \& Van Vleck, 1989; Matos et al., 1996; Costa, 1999; Freitas et al., 2001), entre 0,16 e 0,35.

As médias e os respectivos DP a posteriori e o intervalo de credibilidade da (co)variância genética para PL305 estimada da análise multicaracter (MT) encontram-se na Tabela 4. As maiores estimativas de variância genética foram registradas nos estados de Santa Catarina e Rio Grande do Sul e são inferiores às relatadas por Torres et al. (2000), Rorato et al. (2000) e Araújo et al. (2001), porém, superiores às descritas por Valencia et al. (1998) e Marion et al. (2001).

As estimativas mais precisas da média a posteriori da variância genética foram obtidas nos estados do Paraná e São Paulo, pois foram as que apresentaram os menores DP e as menores amplitudes dos intervalos de credibilidade entre os percentis 2,5 e 97,5\%. Em razão do menor número de registros de lactações analisadas nos estados de Minas Gerais, Santa Catarina e Rio Grande do Sul, era esperado que a precisão das estimativas nestes estados fosse menor. As

Tabela 3 - Médias e desvios-padrão a posteriori e percentis dos componentes de variância, da herdabilidade $\left(h_{a}^{2}\right)$ e da proporção da variância de ambiente permanente $(c)$ para produção de leite $\left(\mathrm{kg}^{2}\right)$ aos 305 dias de lactação

Table 3 - Posterior means and standard deviations, and percentiles of variance components, heritability $\left(h_{a}^{2}\right)$, and permanent environmental variance proportion (c) for 305 day milk yield

\begin{tabular}{lcccc}
\hline $\begin{array}{l}\text { Parâmetro } \\
\text { Parameter }\end{array}$ & $\begin{array}{c}\text { Média } \\
\text { Average }\end{array}$ & \multicolumn{2}{c}{$\begin{array}{c}\text { Percentis (\%) } \\
\text { Percentiles }\end{array}$} \\
\cline { 2 - 4 }$\sigma_{a}^{2}$ & $393.275 \pm 17.724$ & 361.131 & 50 & 97,5 \\
$\sigma_{p}^{2}$ & $255.712 \pm 14.825$ & 226.275 & 392.838 & 428.417 \\
$\sigma_{e}^{2}$ & $767.205 \pm 6.173$ & 755.026 & 255.700 & 282.491 \\
$c$ & $0,181 \pm 0,011$ & 0,159 & 767.131 & 779.475 \\
$h_{a}^{2}$ & $0,278 \pm 0,012$ & 0,257 & 0,181 & 0,199 \\
\hline
\end{tabular}

$\sigma_{a}^{2}=$ variância genética; $\sigma_{p}^{2}=$ variância de ambiente permanente; $\sigma_{e}^{2}=$ variância residual.

$\sigma_{a}^{2}=$ genetic variance; $\sigma_{p}^{2}=$ permanent environmental variance; $\sigma_{e}^{2}=$ residual variance. 
médias a posteriori das covariâncias genéticas entre os estados foram todas positivas e apresentaram DP muito elevados e, em geral, com magnitude muito próxima às próprias estimativas. A covariância genética de maior magnitude foi observada entre os estados de São Paulo e Paraná, o que era esperado, pois foram os estados com maior número de touros em comum e, conseqüentemente, com um dos maiores valores de similaridade genética (Tabela 2). O menor valor para a covariância foi observado entre Paraná e Santa Catarina. As médias a posteriori da variância genética para PL305 estimadas para cada estado, separadamente, foram inferiores às obtidas pelas análises MT, conforme relatado por Falcão et al. (2005), à exceção do Rio Grande do Sul.

As médias, os respectivos DP a posteriori e os intervalos de credibilidade da variância de ambiente permanente $\left(\sigma_{p}^{2}\right)$ para PL305 são apresentados na Tabela 5. As estimativas mais precisas da média posterior de $\sigma_{p}^{2}$ foram as encontradas no Paraná e em São Paulo, estados com menores DP e intervalos de credibilidade de menores amplitudes. A precisão das estimativas de $\sigma_{p}^{2}$ elevou proporcionalmente com o aumento do número de lactações nas análises, assim como ocorreu com as estimativas da variância genética.
Portanto, parece haver um padrão na precisão das estimativas dos componentes de variância, ou seja, a precisão diminui à medida que se reduz o número de lactações.

Houve acentuada diferença na magnitude das médias da variância de ambiente permanente e nas respectivas amplitudes dos intervalos de credibilidade (Tabela 5). As médias posteriores da $\sigma_{p}^{2}$ para os estados de Santa Catarina e Rio Grande do Sul foram, respectivamente, em torno de 4 e 10 vezes maiores que as estimativas REML encontradas por Falcão etal.(2005), em análise unicaracter, provavelmente, em razão da melhoria na estrutura dos dados, propiciada pela análise multivariada.

As médias e os respectivos DP a posteriori e os percentis da variância residual $\left(\sigma_{e}^{2}\right)$ da PL305 estimados com o modelo MT são apresentados na Tabela 6. Para variância residual, também observou-se o mesmo padrão dos demais componentes de variância quanto ao aumento da precisão das estimativas. Os DP a posteriori $\mathrm{e}$ as amplitudes dos intervalos de credibilidade da $\sigma_{e}^{2}$ foram maiores para os estados de Santa Catarina e Rio Grande do Sul. As médias e os respectivos desvios-padrão da variância residual para PL305, obtidos neste trabalho sob o modelo MT, foram menores que as estimativas REML e Bayesianas

Tabela 4 - Médias e respectivos desvios-padrão (DP) a posteriori e percentis da variância $\left(\sigma_{i}^{2}\right)$ e covariância $\left(\sigma_{i, j}\right)$ genéticas para produção de leite $\left(\mathrm{kg}^{2}\right)$ aos 305 dias de lactação, obtidos por análise multicaracter

Table 4 - Posterior means with standard deviations (SD), and percentiles of genetic variance $\left(\sigma_{i}^{2}\right)$ and covariance $\left(\sigma_{i, j}\right)$ for 305 day milk yield, estimated from multiple-trait analyses

\begin{tabular}{|c|c|c|c|c|c|}
\hline \multirow{2}{*}{$\begin{array}{l}\text { Componente de } \\
\text { (co)variância } \\
\text { (Co)variance component }\end{array}$} & \multirow[t]{2}{*}{$\begin{array}{l}\text { Média } \\
\text { Average }\end{array}$} & \multirow[t]{2}{*}{$\begin{array}{c}\mathrm{DP} \\
S D\end{array}$} & \multicolumn{3}{|c|}{$\begin{array}{c}\text { Percentis (\%) } \\
\text { Percentiles }\end{array}$} \\
\hline & & & 2,5 & 50 & 97,5 \\
\hline$\sigma_{1}^{2}$ & 373.928 & 30.005 & 320.120 & 371.185 & 439.239 \\
\hline$\sigma_{1,2}$ & 86.014 & 27.855 & 28.446 & 86.324 & 139.368 \\
\hline$\sigma_{1,3}$ & 75.645 & 29.158 & 16.499 & 74.545 & 129.152 \\
\hline$\sigma_{1,4}$ & 42.369 & 41.086 & -42.013 & 44.476 & 119.512 \\
\hline$\sigma_{1,5}$ & 49.067 & 37.863 & -27.304 & 49.305 & 121.999 \\
\hline$\sigma_{2}^{2}$ & 363.763 & 24.649 & 315.021 & 363.929 & 411.886 \\
\hline$\sigma_{2,3}$ & 131.827 & 23.334 & 71.676 & 133.177 & 173.919 \\
\hline$\sigma_{2,4}$ & 39.139 & 36.043 & -26.960 & 39.675 & 107.799 \\
\hline$\sigma_{2,5}$ & 72.854 & 38.705 & -2.822 & 75.077 & 147.233 \\
\hline$\sigma_{3}^{2}$ & 361.250 & 17.417 & 327.491 & 360.599 & 397.246 \\
\hline$\sigma_{3,4}$ & 36.411 & 35.378 & -37.678 & 35.932 & 102.677 \\
\hline$\sigma_{3,5}$ & 47.811 & 36.283 & -20.332 & 47.450 & 123.196 \\
\hline$\sigma_{4}^{2}$ & 661.855 & 53.534 & 549.107 & 666.098 & 770.598 \\
\hline$\sigma_{4,5}$ & 48.575 & 46.463 & -41.063 & 48.134 & 135.753 \\
\hline$\sigma_{5}^{2}$ & 716.484 & 47.789 & 628.344 & 715.998 & 814.141 \\
\hline
\end{tabular}

i e $\mathrm{j}=1 \ldots 5$, em que $1=\mathrm{MG}, 2=\mathrm{SP}, 3=\mathrm{PR}, 4=\mathrm{SC}$ e $5=\mathrm{RS}$.

$i$ and $j=1 \ldots 5$, where $1=M G, 2=S P, 3=P R, 4=S C$ and $5=R S$. 
Tabela 5 - Médias e respectivos desvios-padrão (DP) a posteriori e percentis da variância de ambiente permanente ( $\sigma_{p}^{2}$ ) para produção de leite $\left(\mathrm{kg}^{2}\right)$, obtidos por análise multicaracter

Table 5 - $\quad$ Posterior means with standard deviations a posteriori of permanent $\left(\sigma_{p}^{2}\right)$ variance for 305 day milk yield, estimated from multiple-trait analyses

\begin{tabular}{|c|c|c|c|c|c|}
\hline \multirow[t]{2}{*}{$\begin{array}{l}\text { Estado } \\
\text { State }\end{array}$} & \multirow[t]{2}{*}{$\sigma_{p}^{2}$} & \multirow[t]{2}{*}{$\begin{array}{l}\mathrm{DP} \\
S D\end{array}$} & \multicolumn{3}{|c|}{$\begin{array}{c}\text { Percentis }(\%) \\
\text { Percentiles }\end{array}$} \\
\hline & & & 2,5 & 50 & 97,5 \\
\hline MG & 293.186 & 27.954 & 233.321 & 296.256 & 343.014 \\
\hline SP & 302.642 & 22.363 & 258.573 & 302.639 & 346.092 \\
\hline PR & 261.565 & 14.660 & 230.846 & 262.800 & 289.571 \\
\hline $\mathrm{SC}$ & 310.830 & 39.051 & 240.264 & 307.946 & 393.486 \\
\hline
\end{tabular}

Tabela 6 - Médias e respectivos desvios-padrão a posteriori e percentis da variância residual ( $\sigma_{e}^{2}$ ) para produção de leite $\left(\right.$ kg ${ }^{2}$ ), estimados por análises multicaracter

Table 6 - Posterior means and standard deviations (SD) a posteriori, and percentiles of residual variance $\left(\sigma_{e}^{2}\right)$ for milk yield estimated from multiple-trait analyses

\begin{tabular}{|c|c|c|c|c|c|}
\hline \multirow[t]{2}{*}{$\begin{array}{l}\text { Estado } \\
\text { State }\end{array}$} & \multirow[t]{2}{*}{$\sigma_{e}^{2}$} & \multirow[t]{2}{*}{$\begin{array}{l}\mathrm{DP} \\
S D\end{array}$} & \multicolumn{3}{|c|}{$\begin{array}{c}\text { Percentis (\%) } \\
\text { Percentiles }\end{array}$} \\
\hline & & & 2,5 & 50 & 97,5 \\
\hline MG & 668.280 & 18.238 & 631.174 & 667.347 & 705.121 \\
\hline SP & 893.599 & 12.383 & 870.834 & 893.812 & 918.644 \\
\hline PR & 666.933 & 7.347 & 652.683 & 667.208 & 681.686 \\
\hline $\mathrm{SC}$ & 712.371 & 23.543 & 666.552 & 712.183 & 759.817 \\
\hline $\mathrm{RS}$ & 815.130 & 27.702 & 762.058 & 814.103 & 869.584 \\
\hline
\end{tabular}

encontradas por Falcão et al. (2005), em análise unicaracter, exceto no Paraná, cujas estimativas foram similares.

As médias de herdabilidade para PL305 foram maiores em Santa Catarina e Rio Grande do Sul, como conseqüência da maior variabilidade genética para PL305 encontrada nestes estados (Tabelas 4 e 7). Os valores de herdabilidade da análise multicaracter encontrados neste trabalho corroboram os descritos na literatura. Freitas et al. (2001) encontraram valores de herdabilidade de $0,16 \pm 0,09,0,17 \pm 0,11 \mathrm{e}$ $0,16 \pm 0,10$ para $1 \stackrel{a}{a}, 2 \underline{a}$ e $3 \stackrel{a}{a}$ lactações, respectivamente, em rebanhos da raça Holandesa em Minas Gerais. Em três regiões do Paraná, os coeficientes de herdabilidade estimados por Rorato et al. (1994) foram $0,68 \pm 0,14,0,28 \pm 0,08 \mathrm{e} 0,33 \pm 02$. Matos et al. (1996) encontraram herdabilidade de 0,35 , ao analisarem lactações de quatro regiões do Rio Grande do Sul. Costa (1999), investigando a heterogeneidade de variância para produção de leite em rebanhos da raça Holandesa, obteve valores de herdabilidade de 0,33 e 0,22 nas classes de baixo e alto desvios-padrão fenotípicos, respectivamente.

As médias e os respectivos erros-padrão a posteriori das correlações genéticas e fenotípicas são apresentados na Tabela 7. As correlações genéticas entre todos os estados foram de baixa magnitude, com o maior valor nos estados de São Paulo e Paraná. Os resultados desta análise sugerem que a produção de leite em cada estado deve ser tratada como característica diferente, como proposto por Falconer(1952).

A baixa magnitude das correlações genéticas entre os pares de estados MG-SC e MG-RS provavelmente reflete os respectivos ambientes contrastantes destas regiões. Os baixos valores de correlações observadas entre PR-SC e PR-RS devem ser decorrentes das diferenças entre os níveis de produção do Paraná, quando comparados com SC e RS. Os fracos laços genéticos devem explicar a baixa magnitude das demais correlações genéticas.

Robertson (1959) estabeleceu, um tanto quanto arbitrariamente, que diferenças biológicas importantes podem ser indicadas quando a correlação genética entre ambientes for menor ou igual a 0,80 . Considerando os baixos valores das correlações genéticas encontradas neste estudo entre as produções de leite em cada estado, era esperado que os animais fossem classificados de forma diferente nos cinco estados.

Vários autores, utilizando registros de lactações de rebanhos da raça Holandesa, têm encontrado altos valores de correlação genética para produção de leite. Carabaño et al. (1989) registraram correlação genética média de 0,81 entre Espanha e Estados Unidos. Sullivan \& Schaeffer (1989) encontraram correlações genéticas entre quatro regiões do Canadá, cujos valores variaram entre 0,85 e 0,89 . Costa et al. (2000) estimaram a correlação genética 
Tabela 7 - Médias e erros-padrão (entre parêntesis) a posteriori da herdabilidade (na diagonal), correlação genética (acima da diagonal) e correlação fenotípica (abaixo da diagonal) para produção de leite em cinco estados, estimados por análise multicaracter

Table 7 - $\quad$ Posterior means and standard erros (parenthesis) of heritability (diagonal), genetic (above) and phenotypic (below) correlation for 305 day milk yield, estimated from multiple-trait analyses

\begin{tabular}{lccrr}
\hline $\begin{array}{l}\text { Estado } \\
\text { State }\end{array}$ & MG & SP & PR & SC \\
\hline MG & $0,280(0,021)$ & $0,234(0,074)$ & $0,206(0,078)$ & $0,086(0,083)$ \\
SP & $0,060(0,019)$ & $0,233(0,015)$ & $0,364(0,064)$ & $0,080(0,074)$ \\
PR & $0,058(0,022)$ & $0,093(0,016)$ & $0,280(0,012)$ & $0,074(0,072)$ \\
SC & $0,028(0,027)$ & $0,024(0,022)$ & $0,025(0,024)$ & $0,393(0,026)$ \\
RS & $0,031(0,024)$ & $0,043(0,023)$ & $0,031(0,030)$ & $0,095(0,073)$ \\
\hline
\end{tabular}

Tabela 8 - Valores da estatística $z$ obtidos para o estudo de diferenças entre os componentes de variâncias genética (acima da diagonal) e residual (abaixo da diagonal) para PL305 entre os cinco estados

\begin{tabular}{|c|c|c|c|c|c|}
\hline $\begin{array}{l}\text { Estado } \\
\text { State }\end{array}$ & MG & SP & PR & $\mathrm{SC}$ & $\mathrm{RS}$ \\
\hline MG & & 0,26 & 0,37 & $-4,69^{*}$ & $-6,07^{*}$ \\
\hline SP & $-10,22^{*}$ & & 0,08 & $-5,06^{*}$ & $-6,56^{*}$ \\
\hline PR & 0,07 & $15,74^{*}$ & & $-5,34^{*}$ & $-6,98^{*}$ \\
\hline $\mathrm{SC}$ & $-1,48$ & $6,81^{*}$ & $-1,84$ & & $-0,76$ \\
\hline RS & $-4,43 *$ & $2,59^{*}$ & $-5,17^{*}$ & $-2,83^{*}$ & \\
\hline
\end{tabular}

${ }^{*} p<0,05$.

entre Brasil e Estados Unidos em 0,85. Entretanto, Rorato (2000) salienta que estudos sobre interação genótipoambiente são contraditórios, tornando necessária a realização de pesquisas nas condições brasileiras.

$\mathrm{Na}$ Tabela 8 encontram-se os valores de $z$ obtidos para o estudo de diferença entre as variâncias genética e residual dos estados analisados. As variâncias genéticas para PL305 foram significativamente diferentes para a maioria dos estados. Entretanto, os valores de $\sigma_{a}^{2}$ não diferiam estatisticamente entre os pares de estados MG-SP, MG-PR e SP-PR. Para as variâncias residuais, as diferenças estatísticas só não foram observadas nos pares de estados MG-PR, MG-SC e PR-SC. Portanto, estes resultados são um indício da existência de heterogeneidade de variâncias genética e residual entre os estados considerados neste estudo.

Os efeitos da heterogeneidade de variância sobre a resposta à seleção dependem das magnitudes das diferenças na variância genética para produção de leite entre os estados e de suas relações com as variâncias fenotípicas. De acordo com os resultados deste estudo, uma das causas da heterogeneidade de variância é de origem ambiental. Portanto, a avaliação genética dos animais pode ser mais influenciada pelo ambiente no qual são criados que pelo seu potencial genético, caso a heterogeneidade de variância seja ignorada.

Várias alternativas foram propostas para se considerar a heterogeneidade de variância nas avaliações genéticas de gado de leite, incluindo o pré-ajustamento dos dados pelo desvio-padrão fenotípico (Wiggans \& VanRaden, 1991). Em estudo conduzido por Ibáñez et al. (1999) para investigar as fontes de heterogeneidade de variância na produção de leite de bovinos da raça Holandesa, o pré-ajustamento dos registros de produção pelo desvio-padrão fenotípico dos grupos contemporâneos, assumindo um modelo de variâncias homogêneas, parece ter corrigido adequadamente a heterogeneidade fenotípica. Todavia, a dispersão dos valores genéticos nos ambientes de maior herdabilidade foi subestimada quando comparada ao modelo de variâncias heterogêneas. Portanto, segundo esses autores, espera-se que o mérito genético das vacas elites dos ambientes de maior herdabilidade sejam subestimados.

Foulley et al. (1990, 1992) e San Cristobal et al. (1993) desenvolveram um procedimento baseado na metodologia dos modelos lineares generalizados (McCullagh \& Nelder, 1989) que permite construir um modelo log-linear para componentes de variância. Esse procedimento possibilita a identificação das fontes causadoras da heterogeneidade de variâncias genética e residual e a quantificação de seus efeitos.

Recentemente, Rekaya et al. (2003) apresentaram o desenvolvimento metodológico de um modelo estrutural (ME), caso particular dos modelos descritos por Foulley et al. (1990, 1992), para estimação das covariâncias genéticas para produção de leite em bovinos da raça Holandesa em cinco regiões dos Estados Unidos. Neste estudo, o ME 
mostrou-se vantajoso nas situações em que existia número limitado de informações para estimação das covariâncias genéticas, principalmente, em razão dos fracos laços genéticos entre as regiões. Outra vantagem importante desta modelagem é a possibilidade de informações externas aos dados serem incorporadas como condições climáticas e como similaridades genética e de manejo entre as regiões, para exploração de padrões nas covariâncias genéticas. As estimativas de covariâncias genéticas e de herdabilidades obtidas pelo ME foram muito mais precisas que as estimadas pelo modelo multicaracter, sem qualquer efeito adverso sobre os valores das correlações genéticas. Ainda segundo os autores, os ME têm menos parâmetros que os modelos multicaracteres, podendo gerar estimativas mais precisas das correlações genéticas.

A presença de heterogeneidade de variâncias genética e residual entre as regiões comprova que essa heterogeneidade não pode ser ignorada nos programas de avaliação genética. Apesar da razoável precisão das estimativas dos componentes de (co)variâncias obtidas neste estudo, a utilização de um modelo estrutural talvez possibilite explorar melhor a estrutura de dados disponível e incorporar informações adicionais para estimação das covariâncias genéticas.

\section{Conclusões}

Os métodos bayesianos via amostrador de Gibbs utilizados neste estudo mostraram-se bastante vantajosos quanto à gama de possibilidades para a exploração das informações disponíveis, principalmente do ponto de vista estatístico. Entretanto, o uso do modelo multicaracter com pouca informação genética disponível e grande quantidade de parâmetros a serem estimados pode ter promovido a baixa precisão das estimativas das covariâncias genéticas.

As estimativas das variâncias genética e residual e de herdabilidade, tanto na análise unicaracter com na multicaracter, foram obtidas com precisão razoável. As precisões destas estimativas estavam associadas ao número de lactações.

Constatou-se a existência de heterogeneidade de variâncias genética e residual para produção de leite entre os cinco estados. Portanto, a heterogeneidade de variância deve ser considerada na avaliação genética dos animais.

Os baixos valores de correlação genética entre os estados evidenciam o efeito da interação genótipo $\times$ ambiente, mostrando que os melhores reprodutores em um estado não o são, necessariamente, nos outros. Portanto, na escolha do reprodutor, a interação genótipo $\times$ ambiente deve ser considerada.

\section{Agradecimento}

À EMBRAPA Gado de Leite e à Associação Brasileira de Criadores de Bovinos da Raça Holandesa, pela cessão dos dados. Aos revisores ad hoc, pelas valiosas sugestões apresentadas para redação final do trabalho.

\section{Literatura Citada}

ARAÚJO, C.V.; TORRES, R.A.; COSTA, C.N. et al. Interação reprodutor $\mathrm{x}$ rebanho na produção de leite da raça Holandesa no Brasil. Revista da Sociedade Brasileira de Zootecnia, v.30, n.3, p.992-999, 2001 (supl. 1).

BOLDMAN, K.G.; FREEMAN, A.E. Adjustment for heterogeneity of variances by herd production level in dairy cow and sire evaluation. Journal of Dairy Science, v.73, n.2, p.503-512, 1990 .

BROTHERSTONE, S.; HILL, W.G. Heterogeneity of variance amongst herds for milk production. Animal Production, v.42, p.297-303, 1986.

CARABAÑO, M.J.; Van VLECK, D.; WIGGANS, G.R. et al. Estimation of genetic parameters for milk and fat yields of dairy cattle in Spain and the United States. Journal of Dairy Science, v.72, p.3013-3022, 1989.

COSTA, C.N. An investigation into heterogeneity of variance for milk and fat yields of Holstein cows in Brazilian herd environments. Genetics and Molecular Biology, v.22, n.3, p.375-381, 1999 .

COSTA, C.N.; BLAKE, R.W.; POLLAK, E.J. et al. Genetic analysis of Holstein cattle populations in Brazil and the United States. Journal of Dairy Science, v.83, p.2963-2974, 2000.

COWLES, K.; BEST, N.; VINES, K. Convergence diagnosis and output analysis. Cambridge: MRC Biostatistics Unit, UK. Version 0.40. 1995.

DE VEER, J.C.; Van VLECK, D.L. Genetic parameters for first lactation milk yields at three levels of herd production. Journal of Dairy Science, v.70, n.7, p.1434-1441, 1987.

DESTEFANO, A.L. Identifying and quantifying sources of heterogeneous residual and sire variance in dairy production data. Ithaca: Cornell University, 1994. 133p. PhD Thesis (Animal Breeding) - Cornell University, 1994.

DONG, M.C.; Van VLECK, L.D. Estimates of genetic and environmental (co)variances for first lactation milk yield, survival, and calving interval. Journal of Dairy Science, v.72, p.678-684, 1989.

DONG, MC.; MAO, I.L. Heterogeneity of (co)variance and heritability in different levels of intraherd milk production variance and of herd average. Journal of Dairy Science, v.73, n.3, p.843-851, 1990.

EVERETT, R.W.; KEOWN, J.F.; TAYLOR, J.F. The problem of heterogeneous within herd error variances when identifying elite cows. Journal of Dairy Science, v.65, p. 100, 1982 (suppl. 1) (Abstract).

FALCÃO, A.J.S.; MARTINS, E.N.; COSTA, C.N. et al. Efeitos do número de animais na matriz de parentesco sobre as estimativas de componentes de variância para produção de leite, usando métodos de máxima verossimilhança restrita e Bayesiano. Revista Brasileira de Zootecnia, 2005 (em tramitação).

FALCONER, D.S. The problem of environment and selection. American Nature, v.86, p.293-298, 1952.

FAMULA, T.R. Detection of heterogeneous variance in herd production groups. Journal of Dairy Science, v.72, n.3, p.715721,1989

FOUlley, J.L.; GIANOLA, D.; SAN CRISTOBAL, M. et al. A method for assessing extent and sources of heterogeneity of residual variances in mixed linear models. Journal of Dairy Science, v.73, n.6, p.1612-1624, 1990. 
FOULLEY, J.L.; GIANOLA, D.; SAN CRISTOBAL, M. et al. Marginal likelihood and Bayesian approaches to the analysis of heterogeneous residual variances in mixed linear gaussian models. Computational Statistics and Data Analysis, v.13, n.3, p.291-305, 1992 .

FreitAS, A.F.; DURÃES, M.C.; VALENTE, J. et al. Parâmetros genéticos para produção de leite e gordura nas três primeiras lactações de vacas Holandesas. Revista Brasileira de Zootecnia, v.30, n.3, p.709-713, 2001.

GIANOLA, D. On selection criteria and estimation of parameters when the variance is heterogeneous. Theoretical Applied Genetic, v.72, p.671-677, 1986.

HENDERSON, C.R. Applications of linear models in animal breeding. Ontario: University of Guelph Press, 1984. 462p.

HILL, W.G.; EDWARDS, M.R.; AHMED, M.K.A. et al. Heritability of milk yield and composition at different levels and variability of production. Animal Production, v.36, p.59-68, 1983.

IBÁÑEZ, M.A.; CARABAÑO, M.J.; ALENDA, R. Identification of sources of heterogeneous residual and genetic variances in milk yield data from the Spanish Holstein-Friesian population and impact on genetic evaluation. Livestock Production Science, n.59, p.33-49, 1999

MARION, A.E.; RORATO, P.R.N.; FERREIRA, G.B. et al. Estudo da heterogeneidade das variâncias para as características produtivas de rebanhos da raça Holandesa no Rio Grande do Sul. Revista da Sociedade Brasileira de Zootecnia, v.30, n.6, p.1995-2001, 2001 (supl. 1).

MATOS, R.S.; RORATO, P.R.N.; FERREIRA, G.B. et al. Parâmetros genéticos para produção de leite e gordura da raça Holandesa no Estado do Rio Grande do Sul. In: REUNIÃO ANUAL DA SOCIEDADE BRASILEIRA DE ZOOTECNIA, 33., 1996, Fortaleza. Anais... Fortaleza: Sociedade Brasileira de Zootecnia, 1996. p.86-87.

McCULLAGH, P.; NELDER, J.A. Generalized linear models. 2.ed. London: Chapman and Hall, 1989. 532p.

MIRANDE, S.L.; Van VLECK, L.D. Trends in genetic and phenotypic variances for milk production. Journal of Dairy Science, v.68, p.2278, 1985.

REKAYA, R.; WEIGEL, K.A; GIANOLA, D. Bayesian estimation of parameters of a structural model for genetic covariances between milk yield in five regions of the USA. In: ANNUAL MEETING EUROPEAN ASSOCIATION FOR ANIMAL PRODUCTION, 50., 1999, Zurich. Proceeding... Zurich: Wageningen Press, 1999. p.6.

REKAYA, R.; WEIGEL, K.A; GIANOLA, D. Bayesian estimation of parameters of a structural model for genetic covariances between milk yield in five regions of the United States. Journal of Dairy Science, v.86, p. 1837-1844, 2003.

ROBERTSON, A. The sampling variance of the genetic correlation coefficient. Biometrics, v.15, p.469, 1959.

ROBERTSON, A.; O'CONNOR, L.K.; EDWARDS, J. Progeny testing dairy bulls at different management levels. Animal Production, v.2, p.141, 1960.
RORATO, P.R.N.; LÔBO, R.B.; MARTINS FILHO, R. et al. Efeito da interação genótipo-ambiente sobre a produção de leite da raça Holandesa, no Estado do Paraná. Revista da Sociedade Brasileira de Zootecnia, v.23, n.5, p.859-869, 1994.

RORATO, P.R.N.; Van VLECK, D.; VERNEQUE, R.S. et al. Interação genótipo-ambiente para a produção de leite em rebanhos da raça Holandesa no Brasil. 2. Uso de um modelo animal. Revista Brasileira de Zootecnia, v.29, n.6, p.20302035, 2000 (supl. 1).

SAN CRISTOBAL, M.; FOULLEY, J.L.; MANFREDI, M. Inference about multiplicative heteroskedastic components of variance in a mixed liner Gaussian model with an application to beef cattle breeding. Genetic Selection Evolution, v.25, p.3-30, 1993.

STATISTICAL ANALYSES SYSTEM - SAS. SAS/STAT User's guide: statistics. 4.ed. versão 8.1, Cary: 2000. v.2.

SULLIVAN, P.G.; SCHAEFFER, L.R. Regional heterogeneity of variances and its effect on Canadian Holstein sire evaluation. Canadian Journal of Animal Science, v.69, p.605-612, 1989.

TORRES, R.A.; BERGMANN, J.A.G.; COSTA, C.N. et al. Ajustamento para heterogeneidade de variância para produção de leite entre rebanhos da raça Holandesa no Brasil. Revista Brasileira de Zootecnia, v.28, n.2, p.295-303, 1999.

TORRES, R.A.; BERGMANN, J.A.G.; COSTA, C.N. et al. Heterogeneidade de variância e avaliação genética de bovinos da raça Holandesa no Brasil. Revista Brasileira de Zootecnia, v.29, n.4, p.1050-1059, 2000.

VALENCIA, E.T.; RAMOS, A.A.; WESCHLER, F.S. et al. Heterogeneidade dos componentes de variância, na produção de leite de rebanhos dos Estados de São Paulo e Paraná, Brasil. In: REUNIÃO ANUAL DA SOCIEDADE BRASILEIRA DE ZOOTECnIA, 35., 1998, Botucatu. Anais...Botucatu: Sociedade Brasileira de Zootecnia, 1998. p.488-490.

Van TASSELL, C.P.; Van VLECK, D.L. A manual for use of MTGSAM. A set of FORTRAN programs to apply Gibbs sampling to animal models for variance component estimation [DRAFT]. Lincoln: U.S. Department of Agriculture, 1995. (Agricultural Research Service), 1995. p.86.

WEIGEL, K.A.; GIANOLA, D.; YANDELL, B.S. et al. Identification of factors causing heterogeneous within-herd variance components using a structural model for variances. Journal of Dairy Science, v.76, n.5, p.1466-1478, 1993.

WIGGANS, G.R.; VANRADEN, P.M. Method and effect of adjustment of heterogeneous variance. Journal of Dairy Science, v.74, n.12, p.4350-4357, 1991.

Recebido: $13 / 02 / 04$ Aprovado:20/10/05 